From Carnegie Mellon University; University of Pittsburgh, Pittsburgh, PA; and Emory University, Atlanta, GA.

Submitted March 16, 2007; accepted September 17, 2007; published online ahead of print at www.jco.org on November 12, 2007.

Supported by funds awarded to the Pittsburgh Mind-Body Center at the University of Pittsburgh and Carnegie Mellon University (Grants No. NIH HL076852 and HL076858), and by National Institutes of Health Grants No. CA104078, NR08272, MH071944, AG024827, AG028015, and AG015321.

Authors' disclosures of potential conflicts of interest and author contributions are found at the end of this article.

Address reprint requests to Michael F. Scheier, PhD, Department of Psychology, Carnegie Mellon University,

Pittsburgh, PA, 15213; e-mail: scheier@ cmu.edu

(C) 2007 by American Society of Clinical Oncology

0732-183X/07/2536-5710/\$20.00

DOI: 10.1200/JCO.2007.11.7093

\title{
Moderators of Interventions Designed to Enhance Physical and Psychological Functioning Among Younger Women With Early-Stage Breast Cancer
}

Michael F. Scheier, Vicki S. Helgeson, Richard Schulz, Suzanne Colvin, Sarah L. Berga, Judy Knapp, and Kristina Gerszten

\section{$\begin{array}{llllllll}\text { A } & \text { B } & \text { S } & \text { T } & \text { R } & \text { A } & \text { C } & \text { T }\end{array}$}

\section{Purpose}

To identify factors that condition or moderate the impact of a previously described set of interventions on psychological and physical adjustment after diagnosis and treatment for earlystage breast cancer.

\section{Patients and Methods}

Younger women (age < 51 years, $N=252$ ) with early-stage breast cancer within 2 months of having completed active nonhormonal adjuvant therapy were randomly assigned to a three-arm clinical trial, consisting of a control arm, an education arm, and a nutrition arm. Primary end points, assessed before random assignment and 4 and 13 months later, included mental functioning, physical functioning, and depressive symptoms. Four types of moderator variables were identified, including two sets reflecting psychosocial resources, specifically personality factors and factors related to the person's social environment, a set reflecting demographic variables, and a set reflecting treatment and disease variables (including comorbidities).

\section{Results}

Psychosocial factors were more likely to moderate treatment effects than were demographic and disease-related factors, but the moderating effects of these psychosocial factors were limited to patients receiving the nutrition intervention. Patients with lower psychosocial resources benefited from the nutrition intervention, whereas patients with a greater amount of psychosocial resources did not.

\section{Conclusion}

Future trials of this type should stratify by or select for the moderating variables identified here (ie, dispositional pessimism, unmitigated communion, and negative social interaction) to establish more firmly their role in responses to psychosocial interventions. Effort should also be made to collect data to inform the delivery of interventions to those who might benefit the most.

\section{J Clin Oncol 25:5710-5714. (C) 2007 by American Society of Clinical Oncology}

\section{INTRODUCTION}

A recently completed clinical trial, ${ }^{1}$ the Breast Cancer Recovery Project (BCRP), has shown that two brief psychosocial interventions, one involving a series of educational sessions and one involving a series of nutritional sessions, can reduce depressive symptomatology and enhance physical functioning among younger women diagnosed with early-stage breast cancer. This article examines whether the main intervention effects previously reported by Scheier et $\mathrm{al}^{1}$ were moderated or conditioned by other factors.

Although one must be cautious when interpreting moderation effects in randomized trials, it is useful to explore moderation effects because they can provide valuable insights about maximizing treatment outcomes. In this regard, past research on moderators suggests that interventions, particularly psychosocial interventions, may have the greatest impact on those persons who are at greatest risk for an adverse outcome. ${ }^{2-5}$ The risk might be a result of maladaptive traits that characterize the person's personality, poor coping skills, or a weak social support network. Regardless of the source of the risk, patients with poorer psychosocial resources seem to benefit more from the interventions than patients with richer psychosocial resources, and we expected the same general pattern of results to emerge here.

Several different types of moderator variables were assessed. One set of variables included personality factors. Of particular interest were optimism ${ }^{6}$ 
(the general tendency to expect positive outcomes in the future) and unmitigated communion $^{7}$ (the tendency to focus on others to the exclusion of oneself to the extent that one's own needs are neglected). Persons more pessimistic in outlook are at heightened risk for a variety of poor health outcomes, ${ }^{8-10}$ as are people who are higher in unmitigated communion. ${ }^{7,11}$ Consistent with their greater risk, pessimistic women with breast cancer have also been shown to benefit more from interventions designed to enhance adjustment. ${ }^{5}$

A second set of moderator variables focused on social environment factors, which have also been linked to differences in adjustment in health-relevant contexts. ${ }^{12-14}$ Included in this set were variables such as the woman's perceptions of perceived support, her level of satisfaction with the relationship she had with her partner, and degree of her family cohesiveness. We also explicitly assessed perceptions of negative social interactions.

A third set of moderators, included for more exploratory reasons, related to the patient's demographic characteristics. Prior research suggests that younger women are at heightened risk for an adverse reaction to diagnosis and treatment for breast cancer compared with older women, ${ }^{15-21}$ although the data are somewhat mixed. ${ }^{22-25}$ This suggests that younger women might benefit more from intervention efforts because they are more vulnerable. In a similar vein, women with a lower socioeconomic status might also benefit more from interventions because they too may be at heightened risk. We included demographic variables in the study to explore these possibilities.

A final set of moderator variables related to the patient's illness, treatment, and general physical health. Included here were factors such as disease severity, extensiveness of treatment, treatment adverse effects, and the number of comorbidities that the patient had. Prior research has shown that treatment and disease factors often have little impact on the psychosocial sequelae of breast cancer. ${ }^{26,27}$ Thus, factors such as these do not seem to put the patient at risk for poor adjustment outcomes. On the basis of these considerations, we expected that treatment- and disease-related variables would not be as likely as personality and social environment variables to emerge as important moderators of intervention effects.

\section{PATIENTS AND METHODS}

\section{Participants}

The data for the present analyses were provided by the women $(\mathrm{N}=252)$ who participated in the BCRP. The participants in the BCRP have been fully described elsewhere. ${ }^{1}$ Eligibility criteria included the following: stage 0 , I, or II breast cancer; no history of treatment for other cancers; age younger than 51 years at the time of recruitment; within 2 months of completion of nonhormonal adjuvant therapy; and English speaking. The study was conducted in accordance with assurances approved by the Department of Health and Human Services and was approved by relevant institutional review boards. All participants signed informed consents.

\section{Protocol}

The protocol used in the BCRP has also been described fully elsewhere. ${ }^{1}$ In brief, participants in the BCRP trial were randomly assigned either to a control arm, which received standard medical care, or to one of two active treatment arms. Each active treatment arm received a series of four group sessions that met once a month for 4 consecutive months. Each monthly session lasted 2 hours. Participants in the education arm received information about their disease and treatment and were provided with a set of relevant coping skills, whereas participants in the nutrition arm received information on how to adopt and adhere to a low-fat, high-fruit, and high-vegetable diet.
Primary outcomes included depressive symptoms (measured by an abbreviated form of the Center for Epidemiologic Studies Depression Scale ${ }^{28}$ ), mental health functioning, and physical health functioning (both quality-oflife variables measured by the Mental Component Summary score and Physical Component Summary score of the Medical Outcomes Study 36-Item Short Form Health Survey, ${ }^{29}$ respectively). Information about primary outcomes was collected at baseline (before random assignment) and 4 months and 13 months later (ie, immediately and 9 months after the intervention, respectively). Information about potential moderators of obtained treatment effects, which provides the focus of the present report, was collected at baseline.

\section{Moderating Variables Assessed}

Personality measures. Dispositional optimism was assessed by the Life Orientation Test-Revised ${ }^{30}$ (mean score $=16.73$, standard deviation $[\mathrm{SD}]=3.78$, range $=1$ to $24, \alpha=.87$; note that all of the descriptive statistics reported for all of the measures come from the present sample). Unmitigated communion was assessed by a nine-item scale created by Fritz and Helgeson ${ }^{31}$ (mean score $=29.72, \mathrm{SD}=5.20$, range $=12$ to $42, \alpha=.75$ ).

Social support/social environment measures. Four social environment variables were assessed. Negative social interaction, which refers to the extent to which the participant was receiving unhelpful support from her social environment, was assessed by five items from the Unsupportive Social Interactions Inventory ${ }^{32}$ (mean score $=7.92, \mathrm{SD}=2.39$, range $=5$ to $16, \alpha=.61$ ). A composite variable measuring partner support satisfaction was created by standardizing and then summing the scores on three separate scales, a 16-item relationship satisfaction measure, ${ }^{1}$ a 16 -item relationship conflict measure ${ }^{1}$ (both developed for this study), and a nine-item measure ${ }^{33}$ assessing the extent to which the partner was accepting of the participant's surgical scars and not reacting in a negative emotional way (mean score $=0.00, \mathrm{SD}=2.62$, range $=-12.38$ to $5.27, \alpha=.82$, for composite measure; average $r$ among components of composite $=0.65$ ). The quality of family interactions was assessed by standardizing and summing the scores on the Cohesion, Conflict, and Expression subscales of the Moos and $\mathrm{Moos}^{34}$ Family Environment Scale (mean score $=0.00, \mathrm{SD}=2.47$, range $=-10.49$ to $5.13, \alpha=.77$, for composite measure; average $r$ among subscales $=0.52$ ). Finally, perceived support was assessed using the six-item version of the Interpersonal Support Evaluation List (mean score $=21.67, \mathrm{SD}=2.95$, range $=10$ to $24, \alpha=.85$ ). ${ }^{35}$

Demographic measures. Demographic measures included age at the time of the baseline interview, marital status, and race/ethnicity. A measure of socioeconomic status was obtained by aggregating across standardized measures of years of education, occupation, and household income.

Disease- and treatment-related measures. Stage of cancer (either 0, I, or II) was used as a marker of disease severity. A treatment extensiveness index was created by standardizing and aggregating type of surgery $(1=$ lumpectomy, $2=$ mastectomy) with type of adjuvant treatment received $(0=$ none, $1=$ radiation or chemotherapy, $2=$ both). Variables were standardized before they were summed. A change in menstrual status between diagnosis and baseline and weight gained or lost since diagnosis (both coded as yes or no) were combined to form an adverse effects of treatment index. In addition, respondents were given at baseline a checklist of potential comorbidities (eg, diabetes, migraines, arthritis, or angina) and were asked to check any conditions that they currently had. The number of conditions checked was used as a measure of comorbidities.

\section{Statistical Analysis}

Moderator variables that were categoric in nature were analyzed using standard analysis of covariance procedures, followed by analyses using simple contrasts. Moderator variables that were continuous in nature were analyzed using regression procedures outlined by Aiken and West. ${ }^{36}$ Variables were centered before they were entered into equations. Also following guidelines provided by Aiken and West, ${ }^{36}$ each moderator was run in two separate equations, one with the nutrition arm dummy coded and one with the education arm dummy coded. Those analyses revealing significant moderator effects were followed by analyses of simple slopes. To analyze the simple slopes, the relations between treatment arms and outcome were evaluated 1 SD above and below the mean of the moderator. 
For those categories of moderator variables in which more than one significant moderator emerged, a subsequent hierarchical regression analysis was conducted in which all significant moderators were entered simultaneously. These analyses also followed the data analytic strategy suggested by Aiken and West. ${ }^{36}$

Because we were interested in moderators of long-term intervention effects, the moderator analyses conducted were limited to treatment outcomes observed at the 9-month postintervention follow-up. Following Aiken and West, ${ }^{36}$ results for continuous moderator variables are reported as unstandardized regression coefficients. Finally, although the interventions did not affect mental health component scores in the original randomized clinical trial, ${ }^{1}$ analyses were conducted to test for moderators of the mental health component. None of the moderators studied here were significant. As a result, mental health functioning will not be discussed further.

\section{RESULTS}

\section{Depressive Symptoms}

Several variables moderated the impact of the nutrition intervention on depressive symptoms. With respect to social support/social environment variables, the effects of the nutrition intervention on depressive symptoms were moderated by perceptions of negative social interaction $\left(B=-0.59, \mathrm{SE}=0.26, \Delta R^{2}=0.02, P<.03\right)$. Calculation of the simple slopes showed that the nutrition intervention significantly lowered depressive symptoms among participants who reported receiving high levels of negative social interaction $(\beta=-.30$, $P<.001)$. The effect of the nutrition intervention among participants who reported experiencing fewer negative social interactions was similar in form but much weaker and nonsignificant $(\beta=-.05, P=.56)$.

The effect of the nutrition intervention on depressive symptoms was also moderated by both personality characteristics, dispositional optimism $\left(B=0.38, \mathrm{SE}=0.17, \Delta R^{2}=0.02, P<.03\right)$ and unmitigated communion $\left(B=-0.24, \mathrm{SE}=0.12, \Delta R^{2}=0.01, P<.05\right)$. With respect to dispositional optimism, analyses of simple slopes indicated that the nutrition intervention significantly reduced depressive symptoms among participants who were more pessimistic in outlook $(\beta=-.31, P<.001)$ but did not have a significant effect on depressive symptoms among those more optimistic in outlook $(\beta=-.05$, $P=.55)$. A conceptually similar pattern emerged for unmitigated communion, in that the nutrition intervention significantly lowered depressive symptoms among those with lower intrapersonal resources (ie, those higher in unmitigated communion; $\beta=-.27, P<.01$ ) but did not affect depressive symptoms among those lower in unmitigated communion $(\beta=-.05, P=.55)$.

To determine the unique contribution of each of these three psychosocial moderator variables, a subsequent hierarchical multiple regression analysis was conducted in which all three moderators were entered simultaneously. Although the effect for negative social interaction approached significance $(P<.09)$, none of the interaction terms attained statistical significance. Thus, it was apparently the shared variance among them that was producing the significant effects in the univariate analyses (even though the average absolute correlation among the moderators was only 0.25 ).

\section{Physical Functioning}

The only variable to moderate intervention effects on physical functioning was comorbidities. Number of comorbidities moderated the impact of both the nutrition intervention $(B=0.83, \mathrm{SE}=0.36$, $\left.\Delta R^{2}=0.02, P<.03\right)$ and the education intervention $(B=-0.71$,
$\left.\mathrm{SE}=0.35, \Delta R^{2}=0.01, P<.05\right)$. Analyses of simple slopes indicated that the nutrition intervention produced a significant improvement in physical functioning among those with more comorbidities $(\beta=.15$, $P<.05)$. In contrast, the nutrition intervention had little impact among patients with fewer comorbidities $(\beta=-.11, P>.18)$. Comorbidities had the opposite effect on the education intervention. Those with fewer comorbidities were benefited by the education intervention $(\beta=.27, P<.001)$, whereas those with more comorbidities were $\operatorname{not}(\beta=.05, P>.49)$.

\section{DISCUSSION}

Scheier et $\mathrm{al}^{1}$ have shown that two brief psychosocial interventions, one involving a series of educational sessions and one involving a series of nutritional sessions, can reduce depressive symptomatology and enhance physical functioning among younger women diagnosed with early-stage breast cancer. The present study compliments this earlier work by identifying who benefited most from those interventions. Several observations can be made with respect to the findings from this study.

First, some participants in the interventions benefited from them, and others did not. More specifically, the effect of the nutrition intervention on depressive symptoms was conditioned by optimism and unmitigated communion, as well as the magnitude of negative social interaction. In addition, comorbidities moderated the impact of both interventions on physical functioning.

Second, the moderation took a form that was generally consistent with prior expectations, in that the interventions were effective for those people who possessed more negative and maladaptive attributes, presumably because they have more to gain from what the interventions have to offer. In this respect, our findings are conceptually consistent with recent resource theories of stress and coping. ${ }^{37-42}$

The nature of the moderators that emerged in this study is noteworthy. That is, both dispositional optimism and social environment factors have been shown in previous studies ${ }^{5}$ to moderate the effects of psychosocial interventions in a manner that is conceptually consistent with the effects that were found here. Taken together, these studies suggest that these factors may play a particularly important role in deciding who might benefit from psychosocial interventions for breast cancer.

It is also important to note, however, that the nature of the interventions that are moderated varies somewhat across studies. Previous research ${ }^{2,3,5}$ has shown that psychosocial factors, such as the ones studied here, can moderate interventions that are similar to the one delivered in the education arm of the present study. Yet, in the present study, the effect of the education intervention was not conditioned by psychosocial factors. It is unclear why these differences across studies have emerged. Perhaps the differences between the present and prior research have to do with differences in the age of the samples studied (the present sample was younger) or in the time at which the interventions were targeted (at the end of nonhormonal adjuvant treatment in the present study $v$ shortly after diagnosis and initiation of treatment in the previous studies). The attempt should be made in future research to better understand these differences in findings.

Why, in the present case, should psychosocial factors be important moderators of outcomes for women in the nutrition arm? One 
possibility is that people with low psychosocial resources eat more poorly and so gain greater psychological benefit from a nutritional rather than an educational intervention. Alternatively, changes to one's eating patterns may be more demanding or harder to enact than the changes called for in the educational intervention. People with low psychosocial resources may have been particularly bolstered by the structure and strategies that the nutrition intervention offered.

The findings with respect to comorbidities were also complex. Prior research ${ }^{43}$ has shown that those with more comorbidities may benefit more from interventions designed to reduce depressive symptomatology than those with fewer comorbidities. We did not find that comorbidities moderated the effects of our interventions on depressive symptoms. However, we did find that comorbidities moderated the effects of our interventions on physical functioning, but the results varied by intervention. With respect to the nutrition intervention, participants with more comorbidities (ie, more burdened or greater need) benefited from the intervention, whereas those with fewer comorbidities did not. This effect is similar conceptually to the general pattern of effects obtained for our psychosocial moderators, as well as the results reported by Reynolds et al. ${ }^{43}$ However, the opposite was true when we examined the effects of the education intervention on individuals with different levels of comorbidities. In this arm, participants with fewer comorbidities benefited.

It is unclear why these differences emerge across outcomes and interventions, but the differences do suggest that the moderating effect of comorbidities is less straightforward than the moderating effects of factors that are more psychosocial in nature. One possibility involves the heterogeneous nature of comorbidities. Perhaps type of comorbidity interacts with the patient population under study, sometimes producing enhanced intervention effects and sometimes producing impaired intervention effects, depending on the specific patient population and the specific comorbidities that are assessed. Future research should be sensitive to this possibility.

Finally, it is of interest to note that the interventions were not moderated by any treatment or disease variable, including extensiveness of treatment and severity of disease. In this regard, it is notewor- thy that conceptually similar results have recently been reported by others. $^{26,27}$ The present findings thus add to the growing body of research that suggests that treatment and disease factors may not be as important as one might think in affecting psychosocial adjustment after breast cancer diagnosis and treatment.

Prior research ${ }^{1}$ has shown that education and nutrition interventions can be created that enhance psychological and physical functioning among younger women with early-stage breast cancer. The present findings add to our understanding by documenting that some women benefit from these interventions and others do not. In general, women benefited if they had poor or maladaptive intrapersonal and/or interpersonal resources. Future trials of this type should stratify by or select for these moderating variables to establish more firmly their role in responses to psychosocial interventions. The information provided by these moderation analyses will help inform the delivery of interventions to those who might benefit the most.

\section{AUTHORS' DISCLOSURES OF POTENTIAL CONFLICTS OF INTEREST}

The author(s) indicated no potential conflicts of interest.

\section{AUTHOR CONTRIBUTIONS}

Conception and design: Michael F. Scheier, Vicki S. Helgeson, Richard Schulz, Suzanne Colvin, Sarah L. Berga, Judy Knapp, Kristina Gerszten Provision of study materials or patients: Michael F. Scheier, Vicki S. Helgeson, Richard Schulz, Sarah L. Berga, Judy Knapp, Kristina Gerszten Collection and assembly of data: Michael F. Scheier, Vicki S. Helgeson, Richard Schulz, Suzanne Colvin

Data analysis and interpretation: Michael F. Scheier, Vicki S. Helgeson, Richard Schulz, Suzanne Colvin

Manuscript writing: Michael F. Scheier, Vicki S. Helgeson, Richard Schulz, Suzanne Colvin, Sarah L. Berga, Judy Knapp, Kristina Gerszten Final approval of manuscript: Michael F. Scheier, Vicki S. Helgeson, Richard Schulz, Suzanne Colvin, Sarah L. Berga, Judy Knapp, Kristina Gerszten

\section{REFERENCES}

1. Scheier MF, Helgeson VS, Schulz R, et al: Interventions to enhance physical and psychological functioning among younger women who are ending nonhormonal adjuvant treatment for early stage breast cancer. J Clin Oncol 23:4298-4311, 2005

2. Helgeson VS, Lepore SJ, Eton DT: Moderators of the benefits of a psychoeducational intervention for men with prostate cancer. Health Psychol 25:348-354, 2006

3. Helgeson VS, Cohen S, Schulz R, et al: Group support interventions for people with cancer: Who benefits from what? Health Psychol 19:107-114, 2000

4. Edgar L, Rosberger Z, Nowlis D: Coping with cancer during the first year after diagnosis. Cancer 69:817-828, 1992

5. Antoni $\mathrm{MH}$, Lehman JM, Kilbourn KM, et al: Cognitive-behavioral stress management intervention decreases the prevalence of depression and enhances benefit finding among women under treatment for early-stage breast cancer. Health Psychol 20:20-32, 2001
6. Scheier MF, Carver CS: Optimism, coping and health: Assessment and implications of generalized outcome expectancies. Health Psychol 4:219-247, 1985

7. Helgeson VS, Fritz HL: A theory of unmitigated communion. Pers Soc Psychol Rev 2:173-183, 1998

8. Scheier MF, Carver CS, Bridges MW: Optimism, pessimism, and psychological well-being, in Chang EC (ed): Optimism and Pessimism: Implications for Theory, Research, and Practice. Washington, DC, American Psychological Association, 2001, pp 189-216

9. Carver CS, Scheier MF: Coping processes and adjustment to chronic illness, in Christensen AJ, Antoni MH (eds): Chronic Physical Disorders: Behavioral Medicine's Perspective. Oxford, United Kingdom, Blackwell, 2002, pp 47-68

10. Scheier MF, Carver CS: Self-regulatory processes and responses to health threats: Effects of optimism on well-being, in Suls J, Wallston K (eds): Social Psychological Foundations of Health. Oxford, United Kingdom, Blackwell, 2003, pp 395-428

11. Helgeson VS: Unmitigated communion and adjustment to breast cancer: Associations and explanation. J Appl Soc Psychol 33:1643-1661, 2003
12. Bloom JR, Stewart $S L$, Johnston $M$, et al: Sources of support and the physical and mental well-being of young women with breast cancer. Soc Sci Med 53:1513-1524, 2001

13. Holland KD, Holahan CK: The relation of social support and coping to positive adaptation to breast cancer. Psychol Health 18:15-29, 2003

14. Brummett $B H$, Babyak MA, Mark DB, et al: Prospective study of perceived stress in cardiac patients. Ann Behav Med 27:22-30, 2004

15. Bloom JR, Kessler L: Risk and timing of counseling and support interventions for younger women with breast cancer. J Natl Cancer Inst Monogr 16:199-206, 1994

16. Bloom JR, Stewart SL, Johnston $M$, et al: Intrusiveness of illness and quality of life in young women with breast cancer. Psychooncology 7:89100, 1998

17. Bloom JR, Stewart SL, Johnston M, et al: General and specific measures of quality of life in younger women with breast cancer, in Baum A, Anderson BL (eds): Psychosocial Interventions for Cancer. Washington DC, American Psychological Association, 2001, pp. 37-56

18. Mor $\mathrm{V}$, Malin M, Allen S: Age differences in the psychosocial problems encountered by breast 
cancer patients. J Natl Cancer Inst Monogr 16:191197, 1994

19. Schnoll RA, Harlow LL, Stolbach $L L$, et al: $A$ structural model of the relationships among stage of disease, age, coping, and psychological adjustment in women with breast cancer. Psychooncology 7:6977, 1998

20. Siegel K, Gluhoski V, Gorey E: Age-related distress among younger women with breast cancer. J Psychosoc Oncol 17:1-20, 1999

21. Wenzel LB, Fairclough $D L$, Brady $M$, et al: Age-related differences in the quality of life of breast carcinoma patients after treatment. Cancer 86:17681774, 1999

22. Baider LA, Kaplan De-Nour A: Breast cancer: A family affair, in Cooper CL (ed): Stress and Breast Cancer. New York, NY, John Wiley and Sons Ltd, 1988, pp 155-170

23. Ganz PA, Lee JJ, Sim MS, et al: Exploring the influence of multiple variables on the relationship of age to quality of life in women with breast cancer. J Clin Epidemiol 45:473-485, 1992

24. Maunsell E, Brisson J, Deschenes L: Psychological distress after initial treatment of breast cancer: Assessment of potential risk factors. Cancer 70:120-125, 1992

25. Goldberg JA, Davidson PM, Murray GD, et al: Psychological morbidity in the first year after breast surgery. Eur J Surg Oncol 18:327-331, 1992

26. Bardwell WA, Natarajan $L$, Dimsdale JE, et al: Objective cancer-related variables are not associated with depressive symptoms in women treated for early-stage breast cancer. J Clin Oncol 24:24202427, 2006
27. Scheier MF, Helgeson VS: Really, disease doesn't matter? A commentary on correlates of depressive symptoms in women treated for earlystage breast cancer. J Clin Oncol 24:2407-2408, 2006

28. Andresen EM, Malmgren JA, Carter WB, et al: Screening for depression in well older adults: Evaluation of a short form of the CES-D. Am J Prev Med 10:77-84, 1994

29. Ware JE, Sherbourne CD: The MOS 36-Item Short Form Health Survey (SF-36): I. Conceptual framework and item selection. Med Care 30:473483, 1992

30. Scheier MF, Carver CS, Bridges MW: Distinguishing optimism from neuroticism (and trait anxiety, self-mastery, and self-esteem): A re-evaluation of the Life Orientation Test. J Pers Soc Psychol 67:1063-1078, 1994

31. Fritz HL, Helgeson VS: Distinctions of unmitigated communion from communion: Self-neglect and overinvolvement in others. J Pers Soc Psychol 75:121-140, 1998

32. Ingram KM, Betz NE, Mindes EJ, et al: Unsupportive responses from others concerning a stressful life event: Development of the Unsupportive Social Interactions Inventory. J Soc Clin Psychol 20:173-207, 2001

33. Wimberly SR, Carver CS, Laurenceau JP, et al: Perceived partner reactions to diagnosis and treatment of breast cancer: Impact on psychosocial and psychosexual adjustment. J Consult Clin Psychol 73:300-311, 2005

34. Moos RH, Moos BS: Family Environment Scale Manual. Palo Alto, CA, Consulting Psychologists Press, 1986
35. Cohen S, Mermelstein R, Kamarck $T$, et al: Measuring the functional components of social support, in Sarason IG, Sarason B (eds): Social Support: Theory, Research and Applications. The Hague, the Netherlands, Martinus Nijhoff, 1985, pp 73-94

36. Aiken LS, West SG: Multiple Regression: Testing and Interpreting Interactions. Newbury Park, CA, Sage Publications, 1991

37. Hobfoll SE: Conservation of resources: A new attempt at conceptualizing stress. Am Psychol 44: 513-524, 1989

38. Hobfoll SE: Stress, Culture, and Community The Psychology and Philosophy of Stress. New York, NY, Plenum Press, 1998

39. Hobfoll SE: The influences of culture, community, and the nested-self in the stress process: Advancing conservation of resources theory. Appl Psychol 50:337-370, 2001

40. Hobfoll SE: Social and psychological resources and adaptation. Rev Gen Psychol 6:307324, 2002

41. Gallo LC, Matthews KA: Understanding the association between socioeconomic status and physical health: Do negative emotions play a role? Psychol Bull 129:10-51, 2003

42. Gallo LC, Bogart LM, Vranceanu AM, et al: Socioeconomic status, resources, psychological experiences, and emotional responses: A test of the reserve capacity model. J Pers Soc Psychol 88:386399, 2005

43. Reynolds CF, Dew MA, Pollock BG, et al: Maintenance treatment of major depression in old age. N Engl J Med 354:1130-1138, 2006

\section{Acknowledgment}

We thank the women who agreed to participate in this project. Without their devotion of time and effort, the information provided by the trial would not have been possible. 